\title{
Das Antriebsmoment Erweiterung und seine Grenzen - Zur Genese und Entwicklungslogik der europäischen Menschenrechts- und Demokratisierungspolitik
}

\author{
Marianne Kneuer*
}

\section{Zur ,Geburtsstunde` europäischer Menschenrechtspolitik in der wissenschaftlichen Debatte}

Die Förderung von Menschenrechten, Demokratie und Rechtsstaatlichkeit ist nominell ein junges Politikfeld der Europäischen Union. Legt man als Voraussetzung für die Existenz eines Politikfeldes die vertragliche Verankerung an, so ist der Vertrag von Maastricht (1993) als Startpunkt zu betrachten, der einen Bezug zur Europäischen Menschenrechtskonvention ${ }^{1}$ und im Bereich der Gemeinsamen Außen- und Sicherheitspolitik (GASP) das Ziel der „Entwicklung und Stärkung von Demokratie und Rechtsstaatlichkeit sowie [der] Achtung der Menschenrechte und Grundfreiheiten“2 enthielt. Frühestens kann die quasi zweifache Erwähnung in der Präambel seit der Einheitlichen Europäischen Akte (1987) hinzugezogen werden. Der qualitative Sprung geschah allerdings erst im Vertrag von Amsterdam, wo erstmals verankert wurde, dass die Union auf den „Grundsätzen der Freiheit, der Demokratie, der Achtung der Menschenrechte und Grundfreiheiten sowie der Rechtsstaatlichkeit" beruht. $^{3}$

Einzuwenden gegen diese Festlegung der ,Geburtsstunde ‘ europäischer Menschenrechtspolitik ist zum einen, dass bereits vor 1993 die einzelnen Organe in verschiedener Form die Förderung von Menschenrechten und Demokratie als Aufgabe formuliert und verfolgt haben. So haben der Rat und der Europäische Rat seit den 1970er Jahren mehrere Deklarationen verfasst. Eckpfeiler sind dabei nicht nur die meist angeführte Menschenrechtsdeklaration des Europäischen Rates von 1991, ${ }^{4}$ sondern bereits die Erklärung über die Menschenrechte (1986), ${ }^{5}$ die Erklärung zur Demokratie $(1978)^{6}$ sowie die Gemeinsame Erklärung von Europäischem Rat, Parlament und Kommission betreffend die Achtung der Grundrechte (1977). ${ }^{7}$ Eine besondere Rolle in diesem Politikfeld nimmt das Europäische Parlament ein, denn es hat bereits sehr früh die Rolle einer Anwältin der Menschenrechte und eines Eintretens für demokratische Prinzipien eingenommen und dieses Profil immer weiter geschärft. Hier lässt sich auf unzählige Stellungnahmen und Deklarationen verwei-

* Prof. Dr. Marianne Kneuer, Professur für Vergleich politischer Systeme/Internationale Beziehungen, Universität Hildesheim.

Ich danke den anonymen Gutachtern für die hilfreichen Hinweise.

1 Art. F Abs. 2 Vertrag über die Europäische Union (EUV) in der Fassung des Vertrags von Maastricht.

2 Art. J.1 Abs. 2 EUV in der Fassung des Vertrags von Maastricht.

3 Art. 6 Abs. 1 EUV in der Fassung des Vertrags von Amsterdam.

4 Erklärung über die Menschenrechte, abgedruckt in: Europa Archiv. Zeitschrift für Internationale Politik 15-16/ 1991, S. D 404-D 406.

5 Erklärung über die Menschenrechte, Brüssel 21. Juli 1986, abgedruckt in: Europa Archiv. Zeitschrift für Internationale Politik 17/1986, S. D 472.

6 Erklärung des Europäischen Rates vom 8. April 1978 (Kopenhagen) Erklärung zur Demokratie, abgedruckt in: Bulletin der Europäischen Gemeinschaften 3/1978, S. 5.

7 Gemeinsame Erklärung des Europäischen Parlaments, des Rates und der Kommission betreffend die Achtung der Grundrechte sowie der Europäischen Konvention zum Schutz der Menschenrechte und Grundfreiheiten, in: Amtsblatt der EG, Nr. C 103 vom 27. April 1977, S. 1-2. 
sen. Eine frühe Wegmarke stellt der Birkelbach-Bericht ${ }^{8}$ der Parlamentarischen Versammlung von 1961 dar, der - so wird hier argumentiert werden - als Keimzelle der europäischen politischen Konditionalität angesehen werden kann und daher eine zentrale Rolle für die Menschenrechts- und Demokratisierungsrolle spielt. Gegen die Geburtsstunde im Vertrag von Maastricht oder Amsterdam spricht zum anderen auch, dass vor 1993 bereits Fakten geschaffen wurden durch die Inkorporierung von Menschenrechtsklauseln in bi- und multilaterale Vertragswerke: so zum Beispiel in die Abkommen mit Albanien 1992 und den Baltischen Staaten 1994, in das AKP-Vertragswerk9 1989 (Lomé IV-Abkommen) sowie in Kooperationsabkommen dritter Generation mit südamerikanischen Ländern ab 1990.

Richtig ist, dass die institutionelle Befassung mit Menschenrechten und Demokratie etwa in Form von Ausschüssen, Abteilungen/Referaten in Generaldirektionen etc. - seit den 1990er Jahren einen Schub erlebt. So wurde das zentrale, vom Europäischen Parlament initiierte Finanzierungsinstrument „Europäische Initiative für Demokratie und Menschenrechte (EIDHR)“ 1994 ins Leben gerufen. Zudem berief es 2004 einen Unterausschuss Menschenrechte ein. In der Kommission erlangte das Thema erst mit der Kommissarin Benita FerreroWaldner (2004-2010) besondere Sichtbarkeit, als sie das Politikfeld „Menschenrechte und Demokratisierung“ im Bereich Auswärtige Beziehungen spezifizierte. ${ }^{10}$ Die Terminologie ist in den einzelnen Organen nicht einheitlich: einmal wird von Menschenrechten und Demokratie, ein anderes Mal von Menschenrechten und Demokratisierung gesprochen. Im Folgenden wird letzterer Begriff benutzt. ${ }^{11}$

Viele wissenschaftliche Untersuchungen fokussieren aufgrund der Vertragslage vornehmlich den Zeitraum seit den 1990er Jahren. Tatsächlich ist die Menschenrechts- und Demokratisierungspolitik der Europäischen Union mit einiger Verzögerung in das Blickfeld der Politikwissenschaft gerückt. Die Zielformulierung im Vertrag von Maastricht, aber auch die sprunghaft gestiegene Rolle insbesondere der Demokratieförderung im Rahmen der postsozialistischen Transformationsprozesse und der Osterweiterung haben dieses Interesse angestoßen. Die Europaforschung hat sich diesem Thema aus verschiedenen Perspektiven zugewandt: in Bezug auf die Untersuchung der spezifisch europäischen Konditionalität im Zuge der Erweiterungspolitik $^{12}$, in der Analyse regionaler EU-Strategien wie in Bezug auf die Mittelmeerpolitik sowie der Europäischen Nachbarschaftspolitik beziehungsweise der Östlichen Partnerschaft. ${ }^{13}$

8 Willi Birkelbach: Bericht im Namen des Politischen Ausschusses über die politischen und institutionellen Aspekte des Beitritts zur Gemeinschaft oder der Assoziierung mit ihr, in: Europäisches Parlament, Sitzungsdokumente 1961/62, 15.01.1962, Dokument 122.

9 AKP-Staaten steht für die Gruppe der afrikanischen, karibischen und pazifischen Staaten.

10 Siehe dazu Europäische Kommission: Furthering Human Rights and Democracy across the Globe, Luxemburg 2007.

11 Es lässt sich einwenden, dass die Termini „Menschenrechts- und Demokratiepolitik“ oder „Menschenrechtsund Demokratisierungspolitik" eine problematische Vermischung zweier zu unterscheidender Politiken darstellen. Da diese Begriffe jedoch von den EU-Organen vorherrschend benutzt werden, werden sie hier in dieser Form ebenfalls aufgegriffen. Siehe beispielhaft Europäische Kommission: Furthering Human Rights, 2007.

12 Siehe etwa Heather Grabbe: The EU's Transformative Power: Europeanization through Conditionality in Central and Eastern Europe, Basingstoke 2006; Geoffrey Pridham: Designing Democracy: EU Enlargement and Regime Change in Post-Communist Europe, Basingstoke 2005; Frank Schimmelfennig/Stefan Engert/Heiko Knobel: International Socialization in Europe: European Organizations, Political Conditionality, and Democratic Change, Basingstoke 2006; Milada Anna Vachudova: Europe Undivided. Democracy, Leverage, and Integration Since 1989, Oxford 2005; Rachel A. Epstein/Ulrich Sedelmaier (Hrsg.): International Influence beyond Conditionality. Post Communist Europe after EU enlargement, London/New York 2009.

13 Siehe etwa Michèle Knodt/Annette Jünemann: Europäische Demokratieförderung durch die Europäische Union, Baden-Baden 2007; Annegret Bendiek: Wie effektiv ist die Europäische Nachbarschaftspolitik? Sechzehn Länder im Vergleich, Stiftung Wissenschaft und Politik: SWP-Studie S 24, Berlin 2008, S. 9; sowie die Arbeiten von Barbara Lippert: Die Europäische Nachbarschaftspolitik: viele Vorbehalte - einige Fortschritte unsichere Perspektiven, Friedrich-Ebert-Stiftung: Internationale Politikanalyse, 2008. 
Innerhalb der jüngeren Literatur zur europäischen Menschenrechtspolitik herrscht eine Kontroverse, die sich auf zwei maßgebliche Positionen kondensieren lässt: Auf der einen Seite wird konstatiert, dass die Europäische Wirtschaftsgemeinschaft in ihren Gründungsverträgen Menschenrechte nicht erwähnt und insofern die Narrative der europäischen Menschenrechtspolitik mit einem ausgedehnten Schweigen begonnen hatte. Später habe die Gemeinschaft dann den Mythos von Menschenrechten und Demokratie als Gründungsprinzip konstruiert. ${ }^{14}$ Die andere Position hebt hervor, dass die Europäische Union das Feld der Menschenrechtspolitik mitnichten mit einem leeren Blatt betrat. ${ }^{15}$ Diese gegensätzlichen Bewertungen lassen sich zumindest teilweise auflösen, wenn man eine wichtige Differenzierung vornimmt, nämlich in eine innere und eine äußere Dimension europäischer Menschenrechtspolitik.

\section{Externalisierung des internen Wertekanons}

Mit innerer Dimension sind die Bereiche von Innen- und Justizpolitik gemeint, die seit dem Vertrag von Amsterdam unter dem Raum der Freiheit, der Sicherheit und des Rechts gefasst werden. Hier ist der Hinweis der Kritiker berechtigt, dass in den 1960er und 1970er Jahren der Europarat die eigentlich herausragende, der Menschenrechtsschutz in der Gemeinschaft dagegen kaum eine Rolle spielte. Das Schweigen der Europäischen Wirtschaftsgemeinschaft/Europäischen Gemeinschaften in der Menschenrechtspolitik wird meist mit dem Fehlen entsprechender vertraglicher Bezüge begründet. Tatsächlich hatte sie kaum Kompetenzen in der Innen- und Justiz- und ebenso wenig in der Außenpolitik. Dennoch entwickelte sich die Menschenrechtspolitik der Gemeinschaft nicht gleichförmig, sondern - so die hier zugrundeliegende Prämisse - zu einem dichotomen Gebilde mit einer Binnendimension, die einen inhärenten Wertekern und dessen vor allem anfänglich stark unterentwickelte Anwendung beinhaltete, und einer Außendimension. Diese äußere Dimension nahm eine andere Entwicklung, insofern bei dem Handeln nach außen Menschenrechte und demokratische Prinzipien als Standards formuliert und angewandt wurden. Letztlich wurde diese Dichotomie erst beendet und die beiden Dimensionen wurden zusammengeführt in der Charta der Grundrechte der Europäischen Union (Grundrechtecharta) und deren Inkorporierung in den Vertrag von Lissabon (2009).

Während innen-, rechts- und außenpolitische Politiken also weder in dem EGKS-Vertrag ${ }^{16}$ noch in den Römischen Verträgen vorhanden waren, steht die freiheitliche, demokratische, rechtsstaatliche und an Menschenrechten orientierte Wertebasis der sechs Gründungsmitglieder außer Frage. Der alleinige Blick auf die Verträge ist daher von begrenzter Aussagekraft: Gerade die Entwicklung des europäischen Außenhandelns zeichnet sich durch eine ,schriftliche Fixierung bereits geübter Verfahrensweisen“17 aus. Allerdings gab es tatsächlich nie einen Masterplan oder ein Konzept für die Förderung von Demokratie, Menschenrechten und Rechtsstaatlichkeit. Vielmehr bildete sich die Menschenrechtspolitik inkremental heraus. Wann lässt sich also jene Externalisierung des gemeinschaftlichen

14 So etwa Andrew Williams: EU Human Rights Policies. A Study in Irony, Oxford 2004, S. 137-140.

15 Vgl. Philip Alston/Joseph H. H. Weiler: An ,Ever closer Union“ in Need of a Human Rights Policy. The European Union and Human Rights, in: Philip Alston (Hrsg.): The EU and Human Rights, Oxford 1999, S. 3-69, hier S. 9.

16 Vertrag zur Gründung der Europäischen Gemeinschaft für Kohle und Stahl.

17 Reinhard Rummel: Zwischen Diplomatenclub und gemeinsamem Entscheidungszentrum. Zukunft der EPZ, in: Reinhardt Rummel/Wolfgang Wessels (Hrsg.): Die Europäische Politische Zusammenarbeit. Leistungsvermögen und Struktur der EPZ, Bonn 1978, S. 297-337, hier S. 317. 
Wertekerns erkennen? Wann und mit welchem Anlass, aus welchen Gründen haben die Europäischen Gemeinschaften begonnen, eine nach außen sichtbare Politik im Hinblick auf Menschenrechte und Demokratie zu formulieren und umzusetzen?

Nur wenige Studien beschäftigen sich mit den ersten Jahrzehnten der Entwicklung der europäischen Menschenrechts- und Demokratisierungspolitik. Dieser Beitrag lenkt den Blick bewusst vor die genannte Wegmarke der Menschenrechtsdeklaration des Europäischen Rates von 1991 und des Vertrags von Maastricht. Die Literatur zu dem lange vernachlässigten Politikfeld Erweiterung ist zwar in den letzten Jahren angewachsen und hat sich auch mit dem Aspekt demokratischer Konditionalität beschäftigt, die Analysen fokussieren derweil überwiegend die Osterweiterung. Diese Lücken sollen hier gefüllt werden. Auf der Grundlage einer historisch-genetischen Betrachtungsweise werden die normativen Grundlagen, die Genese und Entwicklung jener äußeren Dimension der Menschenrechts- und Demokratisierungspolitik nachvollzogen, die eng verbunden mit der Erweiterungs- und Assoziierungspolitik in Erscheinung trat.

In Bezug auf die Frage nach dem Wertekern der Gemeinschaft als Entwicklungsmotiv einer Menschenrechts- und Demokratisierungspolitik lassen sich neo-funktionalistische und konstruktivistische Ansätze verbinden. ${ }^{18}$ Das wiederentdeckte theoretische Erklärungsangebot des revidierten Neo-Funktionalismus, vor allem vertreten durch Philippe C. Schmitter, stellt auf die Externalisierung innerer Handlungsmuster ab, bei dem intern getroffene Vereinbarungen und ,policies“ die Mitglieder zwingen, diese auch als ,collective external position" ${ }^{19}$ an Nichtmitglieder weiterzugeben. Außenpolitische Inhalte - hier: der Menschenrechts- und Demokratisierungspolitik - spiegeln demnach innerhalb der Europäischen Union anerkannte Verhaltensnormen wider ${ }^{20}$ oder anders formuliert: Im Inneren anerkannte und konstitutive Normen werden exportiert. Ähnliches ist mit der Innen-Außen-Analogie gemeint, die als Instrument dienen kann, ,Grundmuster in den Politiken der Union zu entdecken", bei denen im Inneren der Europäischen Union verwirklichte Ordnungsvorstellungen auch ihre Außenbeziehungen anleiten. ${ }^{21}$

Auf der Grundlage dieses Erklärungsansatzes lautet somit das Argument dieser Betrachtung: Die Formulierung demokratischer Prinzipien und das Herausbilden einer Keimzelle von Menschenrechts- und Demokratisierungspolitik wurde induziert im Zuge von externen Herausforderungen, mit denen die Gemeinschaft im Rahmen der Erweiterungs- und Assoziierungspolitik konfrontiert wurde. Die Genese und Entwicklung einer Menschenrechts- und Demokratisierungspolitik lässt sich daher in der Verknüpfung mit der Entwicklung der Erweiterungs- und Assoziierungspolitik erklären. Eine weitere Untersuchungsfrage bezieht sich auf die Innen-Außen-Analogie und inwieweit sich diese ebenfalls in die historisch-genetische Sichtweise einordnen lässt. Sind es diese im Zuge der Erweiterungen jeweils an die Gemeinschaft herangetragenen Herausforderungen, die die Spiegelung der inneren, den Mitgliedern zugrunde liegenden Prinzipien nach außen erzwangen? Dieser Beitrag identifiziert und analysiert die Induktionspunkte, an denen die Formulierung und Anwendung solcher

18 Vgl. Wolfgang Wagner/Gunther Hellmann: Zivile Weltmacht? Die Außen-, Sicherheits- und Verteidigungspolitik der Europäischen Union, in: Markus Jachtenfuchs/Beate Kohler-Koch (Hrsg.): Europäische Integration, Wiesbaden 2006, S. 569-596, hier S. 585.

19 Philippe C. Schmitter: Three Neofunctional Hypotheses About International Integration, in: International Organization 23/1969, S. 161-166, hier S. 165.

20 Ebenda, S. 588.

21 Vgl. Dirk Peters/Wolfgang Wagner: Die EU in den Internationalen Beziehungen, in: Katharina Holzinger/ Christoph Knill/Dirk Peters/Berthold Rittberger/Frank Schimmelfennig/Wolfgang Wagner: Die Europäische Union. Theorien und Analysekonzepte, Paderborn/München/Wien/Zürich, S. 270-272. 
Prinzipien beziehungsweise deren Weiterentwicklung erkennbar werden. Drei zentrale Wegmarken lassen sich ausmachen: Zunächst der Beginn der Erweiterungs- und Assoziierungspolitik in den 1960er Jahren, des Weiteren die Süderweiterung und schließlich die Osterweiterung. Zuletzt werden die Charakteristika der äußeren Dimension der europäischen Menschenrechts- und Demokratisierungspolitik diskutiert und mit einem Blick auf die künftigen Perspektiven verbunden.

\section{Die Keimzelle in den 1960er Jahren - der Birkelbach-Bericht}

Die Erweiterungs- und Assoziierungspolitik stellt einen zentralen Bereich europäischen Außenhandelns dar. Jenseits klassischer außenpolitischer Handlungsformen von Internationalen Organisationen (intergouvernementale Koordination), denen sich die Europäische Wirtschaftsgemeinschaft zudem später zuwendet, verkörpert die Assoziierungs- und Erweiterungspolitik eine Politik sui generis, mit der die Europäischen Gemeinschaften/Europäische Union eine institutionalisierte Verbindung zu einem Drittstaat schafft, deren Qualität über die bekannten Formen partieller oder temporärer Mitgliedschaften in Organisationen hinausgeht. Die Aufnahme weiterer Staaten stellte von Anfang an eine politische Leitidee dar, ein Programm der Gründerstaaten, auch wenn es dazu kein Konzept gab. So zeichnete sich auch die Erweiterung als ein offener Prozess ohne Blaupausen aus. Wenngleich es diesen Titel nicht gab, so bildete die Erweiterungspolitik ein Entstehungs- und Entwicklungsmotiv der Gemeinschaft. ,Letztes und wichtigstes Ziel der Errichtung der Gemeinschaft war von Anfang an stets die politische Einigung Europas [...]", so der Präsident der EWG-Kommission, Walter Hallstein, in seiner Bilanz der Jahre 1961/62.22

Dazu gehörte nicht nur die Erweiterung durch Vollmitgliedschaft. Anfang 1960 wurde die Politik der Assoziierung als „Möglichkeit einer strukturellen Verbindung“ mit den Europäischen Gemeinschaften geschaffen, die als mehr als ein ,bloßer Handelsvertrag“ betrachtet wurde. Die Assoziierung stellte nach dem Willen der damaligen Kommission ein ,,vollwertiges Instrument der europäischen Einigung dar“ “.23 Die Debatten jener Anfangsjahre der Gemeinschaft sind deshalb so aufschlussreich, weil sie die eindeutig politischen Motivationen sowohl von Parlamentarischer Versammlung als auch von Kommission illustrieren, die auf zweierlei abzielten: Zum einen legte man sowohl bei den Beitritts- als auch bei den Assoziierungskandidaten des europäischen Raumes Wert darauf, dass sie bereit sein mussten, „am europäischen Aufbauwerk“ mitzuarbeiten. ${ }^{24}$ So wurde geäußert, dass die Gemeinschaft nur um solche Länder vergrößert werden könne, die nicht nur die wirtschaftliche Seite, sondern auch die politischen Ziele übernehmen. ${ }^{25}$ Nicht gewollt war, dass Länder ,die wirtschaftliche, nicht aber die politische Zielsetzung“ anziehe. ${ }^{26}$ So wurde festgehalten, dass sich Assoziierungsabkommen erübrigten, wenn das Interesse der Kandidaten sich nur auf Handel beziehe. Es bestand sowohl in der Versammlung als auch in der Kommission Konsens darüber, dass die Assoziierung keine rein kommerzielle Angelegenheit sei, sondern auch politische Faktoren enthalte (Jean Rey, Kommission) und, ,daß die EWG kein Handelsunternehmen, sondern eine von Europäern für Europäer geschaffene Gemeinschaft" sei

22 Walter Hallstein: Bilanz, in: Amtsblatt der EWG 12/1962, S. 11.

23 Walter Hallstein in der Debatte des Europäischen Parlaments zur Assoziierung Griechenlands (Bericht Duvieusart), in: Amtsblatt der EWG 11/1961, S. 70-71.

24 Siehe Debatte über Fragen des Beitritts und der Assoziation, in: Bulletin der Europäischen Wirtschaftsgemeinschaft 2/1962, S. 83 (Abgeordneter Dehousse, Belgien) oder S. 84 (Abgeordneter Metzger, Deutschland).

25 Ebenda, S. 84, etwa der Abgeordnete Jarrosson, Frankreich.

26 Ebenda, S. 84-85. 
(Emilio Battista, Vorsitzender Politischer Ausschuss). ${ }^{27}$ Zum anderen spiegeln die Debatten wider, dass demokratische Standards als Leitkriterium aufgefasst wurden. So war den Akteuren seinerzeit zwar bewusst, dass die Gemeinschaft selbst ein demokratisches Defizit in sich trage, dass sie aber dennoch von demokratischen Prinzipien angeleitet werde. ${ }^{28}$

Die Europäische Wirtschaftsgemeinschaft unterschied erstens die Assoziierung mit überseeischen Ländern, zweitens jene mit europäischen Ländern mit einer partiellen Übernahme der politischen Verpflichtungen und drittens schließlich den Beitritt, bei dem sie die vollständige Übernahme des acquis communautaire verlangte. In den 1960er Jahren entwickelte die Europäische Wirtschaftsgemeinschaft somit verschiedene Typen von Assoziierung: zum einen die Beitrittsassoziierungen, wie sie mit Griechenland (1961) und der Türkei (1964) abgeschlossen wurden und zum anderen die Entwicklungs- oder Partnerschaftsassoziierung, wie im Jaunde-Abkommen mit den AKP-Staaten (1963) und den Folgeverträgen sowie in den Abkommen mit den Maghreb-Staaten (1975) und den Maschrik-Staaten (1977), bei denen keine Beitrittsperspektive vorgesehen war. Beiden Typen war gemeinsam, dass sie ein eigenes Rechtsverhältnis begründeten, das die Mitwirkung von Drittstaaten an den Zielen der Gemeinschaft in einer auf Dauer angelegten, institutionalisierten bilateralen und paritätischen Form ermöglichte. ${ }^{29}$ Niederrangige Beziehungen indes stellten etwa Kooperationsund (Frei-)Handelsabkommen dar.

Die Konkretisierung der verschiedenen Formen europäischer Einigung war angestoßen und erforderlich angesichts der Welle von Assoziierungs- und Beitrittsanträgen, die seit 1959 auf die Gemeinschaft traf. 1959 stellten Griechenland und die Türkei Anträge auf Assoziierung, 1961 erreichten die Europäische Wirtschaftsgemeinschaft Beitrittsanträge von Großbritannien, Dänemark und Irland; Österreich, Schweden und die Schweiz zeigten Interesse. 1962 dann beantragte Norwegen den Beitritt und Spanien und Portugal baten um Assoziierung, wobei der Antrag von Francisco Franco das Ziel einer vollen Integration formulierte. Vor diesem Hintergrund sah sich die Gemeinschaft gezwungen, eine Grundlage zu schaffen, wie mit den Anträgen umzugehen sei. Es ging insbesondere darum, künftige Anträge zu kanalisieren, zumal man fürchtete, dass das sehr günstig ausgefallene Abkommen mit Griechenland zusätzliche Interessenten auf den Plan rufen könnte.

Im Oktober 1961 stand daher zur Debatte, entweder eine gemeinsame Politik zu entwickeln, die Bedingungen formuliert, oder im Einzelfall zu entscheiden. Man optierte für die Entwicklung von Kriterien. So wurde im Dezember 1961 eine Kommission gebildet aus Vertretern der Parlamentarischen Versammlung zur Untersuchung der Bedingungen für die Assoziierung und den Beitritt zur Europäischen Wirtschaftsgemeinschaft. Dieser nach dem Berichterstatter benannte Birkelbach-Bericht ${ }^{30}$, wurde im Januar 1962 in der Parlamentarischen Versammlung einstimmig beschlossen und an den Ministerrat weitergeleitet. Er wurde als richtungweisend aufgefasst, als „Doktrin der Europäischen Gemeinschaft““31 Der Bericht legte die geografischen, wirtschaftlichen und politischen Kriterien eines Beitritts fest sowie auch die Fähigkeit, die Verpflichtungen, die sich aus dem Beitritt ergeben, übernehmen zu

27 Ebenda, S. 85

28 Vgl. Jean Monnet: L'Europe unie sera démocratique, in: Bulletin de la CEE 3/1962.

29 Siehe dazu die umfassende Studie von Ulrich Everling: Die Neuregelung des Assoziationsverhältnisses zwischen der Europäischen Wirtschaftsgemeinschaft und den afrikanischen Staaten und Madagaskar sowie den überseeischen Ländern und Hoheitsgebieten, in: Zeitschrift für ausländisches öffentliches Recht und Völkerrecht 24/1964, S. 472-574, hier vor allem S. 525 sowie S. 571-574.

30 Benannt nach dem Berichterstatter Willi Birkelbach, dem deutschen Sozialdemokraten und Vorsitzenden der sozialdemokratischen Fraktion in der Parlamentarischen Versammlung von 1958-1964.

31 Wortbeitrag des belgischen Abgeordneten Duvieusart, Bulletin der Europäischen Wirtschaftsgemeinschaft 2/ 1962, S. 82. 
können. Kernaussage des Birkelbach-Berichts war, dass nur demokratische Staaten zum Beitritt zu den Europäischen Gemeinschaften zugelassen werden sollten, und in gleichem Zuge wurden Bedingungen für die Mitgliedschaft formuliert: „Die Existenzgarantie eines demokratischen Staates im Sinne einer liberalen Organisationsform, ist eine Beitrittsbedingung.“ „Die Länder, deren Regierung die demokratische Legitimierung fehlt und deren Völker nicht an den Entscheidungen der Regierungen teilhaben, sei es direkt oder durch frei gewählte Repräsentanten, können nicht anstreben, in den Kreis der Nationen zugelassen zu werden, die die Europäische Gemeinschaft formen." ${ }^{32}$ Der Bericht entwickelt zwar keinen Kriterienkatalog, nennt aber einige Aspekte, die als Minimum verlangt werden; nämlich dass die Prinzipien des Europarates anerkannt werden, des Weiteren, dass eine demokratische Verfassung besteht. Zudem sollte ein Staat nur dann Mitglied der Gemeinschaft werden, wenn er die grundsätzliche Haltung der Mitgliedstaaten teilt. Trotz des nicht verbindlichen Charakters des Birkelbach-Berichts erhielt gerade dieses politische Kriterium den Status einer Orientierungslinie für die Beurteilung von Beitrittskandidaten. Somit stellt der Birkelbach-Bericht die Keimzelle für die Methode der politischen Konditionalität dar, die die Basis für die dann 1993 durch den Europäischen Rat aufgestellten Kopenhagener Kriterien bildet.

Diese Frühform politischer Konditionalität fand bald ihre ersten Prüfungen, nämlich zum einen aufgrund des Antrags auf Beitrittsassoziierung nach dem griechischen Beispiel von Seiten des autoritären und von Europäischer Wirtschaftsgemeinschaft, NATO ${ }^{33}$ und den europäischen Mitgliedstaaten geächteten Franco-Spaniens 1962 und zum anderen aufgrund des Militärputsches in Griechenland 1967. Franco-Spanien war das erste nichtdemokratische Land, das einen Antrag auf Assoziierung beziehungsweise Beitritt stellte. Deswegen kam der Reaktion der Europäischen Wirtschaftsgemeinschaft darauf eine besondere Bedeutung zu. Der Antrag Spaniens wurde zwar nicht abgelehnt, aber alle Organe - Rat, Kommission und Parlamentarische Versammlung sowie im Übrigen auch einzelne Mitgliedstaaten - sendeten vielfältige Signale, die auf die Bedingung eines demokratischen Wandels hinwiesen. Der Generalsekretär des Rates antwortete dem spanischen Außenminister, ,dass nur nach einer Geste Spaniens im Sinne einer Annäherung seiner politischen Struktur an die Westeuropas eine günstige Berücksichtigung Ihres Antrags möglich ist. “34 In der Parlamentarischen Versammlung gab es eine starke antifranquistische Haltung und der Kommissar für Auswärtige Angelegenheiten, Jean Rey, wies darauf hin, dass die europäische Politik nicht nur von Interessen, sondern auch von Idealen geleitet sei und unterstrich die Vorbehalte in der öffentlichen Meinung und den Widerstand in den nationalen Parlamenten der Mitgliedstaaten. ${ }^{35}$

Nachdem Franco im Juni 1962 spanische Teilnehmer eines Kongresses der Europabewegung in München mit harter Repression bestrafte, ließen die entsprechenden Reaktionen nicht lange auf sich warten: Die drei europäischen Parteienfamilien und die Versammlung selbst verabschiedeten Resolutionen, nach denen ein Land, das nicht demokratische Prinzipien vertrete, nicht der Gemeinschaft beitreten könne. ${ }^{36}$ Auf wiederholte Nachfrage der

32 Rapport fait au nom de la commission politique sur les aspects politique et institutionnels de l'adhésion ou de l'association à la Communauté par M. Willi Birkelbach Rapporteur (19.12.1961), in: Assemblé parlamentaire européenne, Documents de séance 1961-1962, Document 122. Die Übersetzung wurde von der Autorin vorgenommen.

33 North Atlantic Treaty Organization.

34 Brief vom 14.03.1962, Ministerio des Asuntos Exteriores, R 6916/E 7, zitiert nach Maria Teresa La Porte: La política europea del régimen de Franco. 1957-1962, Pamplona 1992, S. 365.

35 Brief Jean Rey, Ministerio de Asuntos Exteriores, R 6915E 65, zitiert nach La Porte: La política europea del régimen de Franco, 1992, S. 370.

36 Vgl. Europäisches Parlament: Sixtième rapport sur l'activité de la Communauté (01.05.1961-30.04.1962), Annex Institutions. 
spanischen Regierung entschied sich der Rat 1967 zu Verhandlungen über ein Präferenzabkommen, die nach weiteren drei Jahren zum Abschluss eines letztlich mageren Handelsabkommens führten. Die offizielle Haltung und die Aktionen der Gemeinschaftsinstitutionen zwischen 1960 und dem Ende des Franco-Regimes, auch noch während der Transition, machten sowohl der politischen Elite als auch der Bevölkerung klar, dass die Demokratisierung eine Bedingung für den Eintritt in die Europäischen Gemeinschaften war.

Ein weiterer Beleg für die Unvereinbarkeit nichtdemokratischer Regierungsformen und einer Beitrittsassoziierung war, dass die Gemeinschaft 1967 auf den Obristenputsch in Griechenland mit dem Einfrieren der meisten Bestimmungen reagierte (Ausnahme: Zollunion). Dieser Status blieb bis zur Wiederherstellung der zivilen Regierung 1974 bestehen. So gab es auch keinen Fortschritt bei der - ursprünglich beabsichtigten - Harmonisierung der Agrarpolitik. Die Konsequenzen waren erheblich, und zwar nicht nur in finanzieller Hinsicht. Innerhalb der internationalen Missbilligung der Militärjunta war die Rolle der Gemeinschaft von herausgehobener Bedeutung. Wie im Falle Spaniens machten die Demarchen der Parlamentarischen Versammlung und die offiziellen Äußerungen von Kommissions- oder Ratsmitgliedern eindeutig klar, dass keine Verbesserung in den bilateralen Beziehungen zu erwarten sei, solange Griechenland unter militärischer Führung stehe. Diese von den Grundsätzen politischer Konditionalität geprägte Haltung der Europäischen Gemeinschaften spielte eine erhebliche Rolle für die demokratische Opposition in Spanien und Griechenland, die sich bestärkt fühlte und einen wichtigen externen Akteur hinter sich sah. ${ }^{37}$

Es lässt sich in den 1960er Jahren somit ein entscheidender Prägefaktor erkennen: Die Politik, die Menschenrechts- und Demokratiestandards formuliert und außenpolitischen Entscheidungen zugrunde legt, wurde im Zuge der Erweiterung und Assoziierung der Europäischen Gemeinschaften generiert und zwar in Form der politischen Kriterien. Zugleich lassen sich aus den Reaktionen der Gemeinschaftsorgane auf politische Verfehlungen, demokratische Rückschläge oder undemokratische Regierungen die Formen von Sanktionen erkennen. Dies gilt allerdings nur im Hinblick auf potenzielle Beitrittskandidaten. Die Entwicklungs- oder Partnerschaftsassoziierung ließ solche politischen Kriterien außen vor und kannte daher auch keine steuernden Elemente in Bezug auf die Menschenrechtssituation oder die Regierungsform der Partnerländer, etwa der AKP-Staaten. Das heißt, es konstituierte sich eine hinsichtlich ihrer Menschenrechts- und Demokratieansprüche dichotome Politik der Europäischen Gemeinschaften: Bedingungen und Sanktionen bei Nichterfüllung bezüglich eines Beitritts beziehungsweise einer Beitrittsassoziierung einerseits und das Fehlen politischer Kriterien bei der Nichtbeitrittsassoziierung andererseits. Diese Dichotomie der Menschenrechts- und Demokratisierungspolitik ist nicht deckungsgleich mit dem Dualismus, der bezogen auf die vergemeinschafteten Felder des Außenhandelns, wie die Handelspolitik, und der noch intergouvernemental gestalteten Außenpolitik (GASP) besteht. Es handelt sich um eine Dichotomie, die bei der Verankerung und Durchsetzung von politischen Kriterien zugrunde liegt und die zu unterschiedlichen Ansätzen einmal bei der Erweiterungspolitik und zum anderen bei allen anderen bi- oder multilateralen Partnerschaften ohne Beitrittsperspektive führt.

37 Für Griechenland vgl. Susannah Verney/Theodore Couloumbis: „State-International Systems Interaction and the Greek Transition to Democracy in the mid-1970s“, in: Geoffrey Pridham (Hrsg.): Encouraging Democracy. The International Context of Regime Transition in Southern Europe, Leicester 1991, S. 103-125. Für Spanien vgl. Marianne Kneuer: Demokratisierung durch die EU. Süd- und Ostmitteleuropa im Vergleich, Wiesbaden 2007, S. 162-174. 
Die kumuliert auftretenden Beitrittsaspirationen, aber auch die bevorstehende erste Erweiterung der Gemeinschaft schärften nicht nur das Bewusstsein für die politischen Requisiten, sondern auch für die identitätsbildende gemeinsame Wertebasis. Obwohl die erste Erweiterung angesichts der Beitrittskandidaten in Bezug auf Menschenrechts- oder Demokratiestandards völlig unproblematisch war, scheint die Verbindung zwischen der Identität Europas und der Respektierung von Freiheiten und der Rekurs auf die frei gewählten Parlamente als Voraussetzung nicht zufällig, wie es der Bericht der Außenminister 1970 an die Staats- und Regierungschefs ausdrückt: „Das geeinte Europa muß [!] auf dem gemeinsamen Erbe der Achtung der Freiheit sowie der Menschenrechte fußen und demokratische Staaten mit frei gewählten Parlamenten in sich vereinigen. “38 Mit dem ,Dokument über die europäische Identität" von 1973 wurde die gemeinsame Wertebasis als Beitrittskriterium aufgegriffen: „Das europäische Einigungswerk [...] steht den anderen europäischen Nationen offen, die die Wertvorstellungen und Ziele der Neun teilen. "39 Erstmals wird hier jener bislang überwiegend implizite Wertekern explizit formuliert. Aufschlussreich ist, dass beide Dokumente der frühen 1970er Jahre die Bestimmung ihrer Identität und des demokratischen Kerns im Kontext mit neuen außenpolitischen Zielen sehen, nämlich mehr Verantwortung in der Welt zu übernehmen und die Beziehungen zu den übrigen Ländern der Welt näher zu definieren.

\section{Wegmarke Süderweiterung - politische Konditionalität im Test}

Der zweiten, vergleichsweise freilich größeren Herausforderung für ihre Erweiterungsund Demokratisierungspolitik sah sich die Gemeinschaft im Zuge der demokratischen Transitionen von Griechenland, Portugal und Spanien gegenüber, denn die südeuropäischen Länder stellten zwischen 1975 und 1977 Beitrittsanträge. Zwar gab es erste Erfahrungen mit der Erweiterung, aber nicht mit der Aufnahme von Transitionsländern. Die politische Botschaft der Europäischen Gemeinschaften an die südeuropäischen Länder bestand in der konsequenten Anwendung der politischen Konditionalität des Birkelbach-Berichts. Man gab der Hoffnung auf eine demokratische Entwicklung des Landes Ausdruck, eröffnete die Perspektive auf eine Mitgliedschaft bei vorheriger Erfüllung der Bedingungen. Die Europäischen Gemeinschaften machten deutlich, dass das Ende der griechischen Militärdiktatur, der Sturz Marcello Caetanos in Portugal und der Tod Francos in Spanien allein nicht ausreichten für einen Beitritt; klare Belege für Demokratisierung wurden gefordert. ${ }^{40}$ Dabei verfolgten und beurteilten alle Organe, insbesondere aber die Parlamentarische Versammlung, sehr genau die Reformschritte in den Ländern, was durchaus auch in Hinweisen auf mangelnde demokratische Standards münden konnte. Bei Rückschritten wurde direkt mit sehr konkreten Empfehlungen reagiert. Dies war insbesondere bei Portugal und Spanien der Fall.

In Portugal herrschten zwischen 1974 und 1976 zunächst instabile Verhältnisse, was dazu führte, dass der Europäische Rat zwar Soforthilfemaßnahmen bewilligte, aber eine substanzielle bilaterale Annäherung nicht vorankam. Die Europäischen Gemeinschaften waren erst zu einem weitergehenden Abkommen bereit bei der Erfüllung bestimmter Elemente einer

38 Erster Bericht der Außenminister an die Staats- und Regierungschefs der EG-Mitgliedstaaten vom 27. Oktober 1970 (Luxemburger Bericht), Erster Teil, Punkt 5, in: Presse- und Informationsamt der Bundesregierung, Texte zur Europäischen Politischen Zusammenarbeit (EPZ), Bonn 1974, S. 17-25.

39 Dokument über die europäische Identität, Bulletin der Europäischen Gemeinschaften 12/1973, S. 118-122, hier Punkt 4.

40 Für Griechenland vgl. Verney/Couloumbis: State-International Systems Interaction, 1991, S. 117; für Portugal vgl. Christopher Preston: Enlargement and Integration in the European Union, London/New York 1997, S. 67; für Spanien siehe Kneuer: Demokratisierung durch die EU, 2007, S. 204-210. 
pluralistischen Demokratie. ${ }^{41}$ Im Falle Spaniens kritisierten die Organe das Reformgesetz der spanischen Regierung von 1976 scharf, da die dort vorgesehene Stellung des Parlamentes nicht die „demokratischen Requisiten erfüll[t], die die Demokratien Westeuropas sich selbst setzen." ${ }^{2}$ Angemahnt wurden die Herstellung der individuellen und politischen Rechte, die Anerkennung der Gewerkschaften, die baldige Legalisierung aller politischen Parteien ohne Ausnahme und eine Generalamnestie, die auch den Exilanten die Rückkehr ermögliche. Ein zentraler Aspekt war in Spanien insbesondere die Zulassung der kommunistischen Partei, die nicht nur für das Parlament, sondern auch für den Rat Bedingung war. Schließlich galt für alle Kandidaten, dass demokratische Wahlen die Voraussetzung für einen Beitrittsantrag darstellten. In dieser Phase bis zu den jeweiligen ersten Wahlen spielten die Europäischen Gemeinschaften eine deutlich aktivere und auch stärker intervenierende Rolle in Bezug auf Menschenrechts- oder Demokratiestandards, als dies bei bilateralen Beziehungen üblich war. Die Anforderungen wurden sehr viel konkreter formuliert und bezogen sich auf die verfassungsrechtliche Garantie der Grundfreiheiten und eines uneingeschränkten gesellschaftlichen Pluralismus.

Die Demokratisierung der drei südeuropäischen Staaten und deren dezidierter Beitrittswille katalysierten die in dem „Dokument zur europäischen Identität“ begonnene Rollendefinition der Europäischen Gemeinschaften. Zwei Aspekte kristallisierten sich dabei heraus: Zum einen waren sich die Europäischen Gemeinschaften ihrer moralisch-politischen Verantwortung gegenüber den jungen Demokratien des Südens bewusst und bereit, diese zu übernehmen. So formulierte die Kommission mit Verweis auf die Präambel des Vertrags von Rom, in ihren „Überlegungen zur Erweiterung der Gemeinschaft“" vom April 1978: „Die drei Länder haben der Gemeinschaft eine politische Verantwortung übertragen, der sie sich nur entziehen könnte, wenn sie selbst die Grundsätze ihres eigenen Aufbaus verleugnet." 43 Zum anderen schärfte sich das Bewusstsein als eine demokratische Gemeinschaft, die drei ehemalige Diktaturen aufnimmt und diesen einen Konsolidierungsrahmen bietet, um sie vor einem Rückfall in die Diktatur zu schützen, wie in demselben Dokument formuliert wurde.

In diesem Zusammenhang ist auch die „Gemeinsame Erklärung von Europäischem Parlament, Rat und Kommission“ von $1977^{44}$ zu sehen, der die „Erklärung zur Demokratie“ des Europäischen Rates von $1978^{45}$ folgte. Als Anlass ist hier der bevorstehende griechische Beitritt zu sehen: nicht nur, dass Griechenland das erste Land einer Erweiterungsrunde mit nichtdemokratischem Hintergrund war, sondern die Gemeinschaft hatte mit Griechenland in den 1960er Jahren bereits einmal einen Rückfall erlebt. In der Gemeinsamen Erklärung wird die herausragende Bedeutung unterstrichen, die dem Schutz der Menschenrechte - abgeleitet von den einzelnen Verfassungen der Mitgliedstaaten sowie der Europäischen Konvention für Menschenrechte und Grundfreiheiten - beigemessen wird. Ausdrücklich wird darauf hingewiesen, dass alle Mitgliedstaaten Zeichner der Menschenrechtskonvention sind. ${ }^{46}$ Der

41 Vgl. Frances Nicholson/Roger East: From the Six to the Twelve. The enlargement of the European Communities, Essex 1987, Kap. 11.

42 So der Berichterstatter des Europäischen Parlaments, der französische Sozialist Maurice Faure, in seinem Bericht vom 12.05.1976.

43 Mitteilung der Kommission an den Rat: Erweiterung der Gemeinschaft. Umfassende Überlegungen, in: Bulletin der Europäischen Gemeinschaften 1/1978, 20.04.1978, S. 6-18, hier S. 6.

44 Gemeinsame Erklärung des Europäischen Parlaments, des Rates und der Kommission betreffend die Achtung der Grundrechte, 1977.

45 Erklärung des Europäischen Rates vom 8. April 1978 (Kopenhagen) Erklärung zur Demokratie, 1978.

46 Gemeinsame Erklärung des Europäischen Parlaments, des Rates und der Kommission betreffend die Achtung der Grundrechte, 1977. 
Europäische Rat verdeutlichte mit der Demokratieerklärung ebenfalls, dass ,,die Aufrechterhaltung der parlamentarischen Demokratie und der Menschenrechte in allen Mitgliedstaaten wesentliche Elemente ihrer Zugehörigkeit zu den Europäischen Gemeinschaften sind. “47 Auch die erstmalige und dazu noch doppelte Erwähnung in der Präambel der Einheitlichen Europäischen Akte (1987) weist auf diesen Zusammenhang mit der Süderweiterung und auf die Fortentwicklung der Dokumente der 1970er Jahre hin, die den demokratischen Kern der Gemeinschaft und den Schutz der Menschenrechte absichern sollten. Es wird einmal die Entschlossenheit, ,gemeinsam für die Demokratie einzutreten“, Menschenrechte, Grundfreiheiten und die in der Europäischen Sozialcharta anerkannten Grundrechte zu schützen, ausgedrückt und zum zweiten bekennt sich die Gemeinschaft zum Eintreten „ganz besonders für die Grundsätze der Demokratie und die Wahrung des Rechts und der Menschenrechte“. 48

Zwar blieben die Europäischen Gemeinschaften vor dem demokratischen Rückfall eines Mitgliedstaates verschont, dennoch hätten sie damals wohl trotz der Demokratieerklärung Schwierigkeiten gehabt darauf zu reagieren, denn Sanktionen oder ein Ausschluss etwa waren vertraglich nicht vorgesehen. So sah es ein damaliger Berater der Kommission als selbstverständlich an, dass die Errichtung einer Volksdemokratie oder einer Rechtsdiktatur in einem Mitgliedstaat ,zwangsläufig' zur Folge haben müsse, dass seine Rechte und Pflichten suspendiert werden (zum Beispiel keine Zahlungen aus den Fonds oder von der Europäischen Investitionsbank). Zugleich weist er darauf hin, dass der EWG-Vertrag keine ausdrücklichen Vorschriften für so einen Fall vorsehe und fordert deswegen eine rechtlich verbindliche Erklärung; und zwar nicht nur im Hinblick auf die drei südeuropäischen Kandidaten, sondern auch mit Rücksicht auf die Mitgliedstaaten, in denen kommunistische Parteien, deren Demokratie-Verständnis keinesfalls über alle Zweifel erhaben sei, nach der Macht strebten. ${ }^{49}$ Solch ein Mechanismus, mit dem ,eine anhaltende und schwerwiegende Verletzung“ der Grundsätze von Freiheit, Demokratie, Achtung der Menschenrechte und Rechtsstaatlichkeit geahndet werden kann in Form von Aussetzung bestimmter Rechte, einschließlich der Stimmrechte, wurde jedoch erst im Vertrag von Amsterdam eingebaut. ${ }^{50}$ Hintergrund dieses Sanktionsmechanismus in Art. 7 EUV in der Fassung des Vertrags von Amsterdam, der sich auch in der Fassung des Vertrags von Lissabon findet, war wiederum eine bevorstehende Erweiterung, nämlich die um die postsozialistischen Staaten.

\section{Wegmarke Osterweiterung - Verfeinerung der Kriterien}

Ähnlich wie bei der Süderweiterung handelte es sich bei der Osterweiterung um eine neue und präzedenzlose Erweiterung, nicht nur im Hinblick auf die Quantität der Kandidaten (und die wirtschaftlichen Implikationen, die hier ausgespart bleiben), sondern auch was den notwendigen Konsolidierungsstand der Demokratien anging. Die Methode der Konditionalität, die bereits in den 1960er Jahren und im Vorfeld der Süderweiterung praktiziert worden war, prägte direkt nach 1989 auch die Politik der Gemeinschaft gegenüber den ostmitteleuropäischen Transformationsstaaten. Die drei wichtigsten Elemente der EG-Politik gegenüber den Ländern Ostmitteleuropas in der Anfangsphase - das Unterstützungspro-

47 Erklärung des Europäischen Rates vom 8. April 1978 (Kopenhagen) Erklärung zur Demokratie, 1978.

48 Präambel der Einheitlichen Europäischen Akte.

49 Vgl. Eberhard Rhein: Plädoyer für eine erweiterte Gemeinschaft, in: Hajo Hasenpflug/Beate Kohler: Die SüdErweiterung der Europäischen Gemeinschaft. Wende oder Ende der Integration?, Hamburg 1977, S. 49-60, hier S. 52.

50 Art. 7 EUV in der Fassung des Vertrags von Amsterdam. Diese Möglichkeit wurde auch in Art. I-59 des Vertrags über eine Verfassung für Europa (Verfassungsvertrag) festgeschrieben. 
gramm PHARE ${ }^{51}$ die Europäische Bank für Wiederaufbau und Entwicklung (EBWE) und die Europa-Abkommen - enthielten die Bindung an demokratische und marktwirtschaftliche Prinzipien. ${ }^{52}$ Die Bedingung für finanzielle und wirtschaftliche Hilfe bestand nicht nur in der Reform hin zu Marktwirtschaften, sondern auch in Fortschritten bei der politischen Demokratisierung. So setzte der Rat der Außenminister bei seinem Treffen im Februar 1990 fünf Prinzipien fest als Vorbedingungen zur Unterzeichnung der Assoziierungsabkommen, von denen sich neben der Entwicklung einer marktorientierten Wirtschaft vier auf den politisch-rechtlichen Bereich bezogen: Schaffung einer Rechtsordnung, Respektieren der Menschenrechte, Einführung einer Mehrparteiendemokratie, Abhalten freier, fairer und kompetitiver Wahlen. Die Einhaltung dieser Prinzipien wurde durch die Kommission im März 1990 vor Ort überprüft. Erst nach der positiven Überprüfung dieser Kriterien wurden die Hilfsprogramme auf Bulgarien, die Tschechoslowakische Republik, die DDR und Jugoslawien ausgedehnt. Beim Ausschluss Rumäniens wurde wieder die politische Konditionalität erkennbar: Die sich verschlechternde Menschenrechtssituation war Anlass für den Ausschluss aus PHARE und für die Nichtunterzeichnung des Handels- und Kooperationsabkommens, das die Kommission im Juni 1990 auszuhandeln begonnen hatte. ${ }^{53}$ Mit dieser sehr frühen Verpflichtung auf einen demokratischen Weg und die Sanktionen setzten die Europäischen Gemeinschaften klare Signale.

Auch bei den Assoziierungsabkommen (Europa-Abkommen) wurden die politischen Requisiten festgelegt. ${ }^{54}$ Bei den Europa-Abkommen handelt es sich um ein ähnliches Instrument, wenngleich deutlich umfassender, wie bei der Beitrittsassoziierung mit Griechenland und der Türkei in den 1960er Jahren: Zwar konnten sich die Europäischen Gemeinschaften bei Unterzeichnung nicht dazu entschließen, eine Beitrittszusage aufzunehmen, dennoch wurden die Verträge als ,besondere Form der Assoziierung betrachtet“, mit der die Länder an die Europäischen Gemeinschaften herangeführt werden sollten. Die Europa-Abkommen, die zunächst 1991 mit Polen, Ungarn und der Tschechoslowakischen Republik (nach der Auflösung neu unterzeichnet) bis 1995 auch mit Bulgarien, Rumänien, Slowenien und den Baltischen Staaten abgeschlossen wurden, nennen pluralistische Demokratie, Rechtsstaatlichkeit, Achtung der Menschenrechte und Grundfreiheiten, Mehrparteiensystem und freie und demokratische Wahlen als Kriterien. ${ }^{55}$ Dabei war nicht nur die Aufnahme einer Menschenrechts- und Demokratieklausel in die Verträge eine Innovation, sondern auch die sogenannte Baltikum-Klausel, nämlich die in den ersten Abkommen mit den Baltischen Staaten, Albanien und Slowenien aufgenommene Zusatzklausel, die die Suspendierung bei Nichterfüllung enthielt.

51 Die Abkürzung PHARE steht für „Poland and Hungary: Aid for Restructuring of the Economies“. Das Programm wurde später auf weitere Staaten ausgedehnt.

52 Die Statuten der EBWE beinhalten die Verpflichtung zu und Anwendung der Prinzipien eines Mehrparteiensystems, Pluralismus und Marktwirtschaft. Vgl. dazu auch John Pinder: The European Community and democracy in Central and Eastern Europe, in: Geoffrey Pridham/Eric Herring/George Sanford (Hrsg.): Building democracy? The international dimension of democratisation in Eastern Europe, New York 1997, S. 119-144, hier S. 124.

53 Erst im Januar 1991 wurde Rumänien wieder in das PHARE-Programm aufgenommen.

54 Vgl. dazu Barbara Lippert/Heinrich Schneider (Hrsg.): Monitoring Association and Beyond. The European Union and the Visegrad States, Bonn 1995; sowie Heather Grabbe: A Partnership for Accession? The Implications of EU Conditionality for the Central and East European Applicants, European University Institute: EUI Working Papers RSC No. 99/12, San Domenico 1999.

55 Vgl. Präambel der Europa-Abkommen. Siehe beispielhaft Gesetz zu dem Europa-Abkommen vom 16. Dezember 1991 zur Gründung einer Assoziation zwischen den Europäischen Gemeinschaften und ihren Mitgliedsstaaten und der Republik Ungarn, Bundesgesetzblatt 1993, Teil II, S. 1472-1715. Die Verträge sind alle analog aufgebaut. 
Es lässt sich zudem - wie bereits bei den beiden besprochenen Wegmarken - erkennen, dass die Auseinandersetzung mit dem Politikfeld Menschenrechte und Demokratie einen neuerlichen Schub erfuhr. Die einzelnen Organe verfassten entsprechende Dokumente, die sich der Förderung verpflichteten - auch über die möglichen post-sozialistischen Beitrittskandidaten hinaus: so die Menschenrechtsdeklaration des Europäischen Rates von 1991, die beiden Mitteilungen der Kommission von 1995, die Verordnungen des Rates zur Implementierung der Ziele - Entwicklung und Konsolidierung von Demokratie, Rechtsstaatlichkeit und Menschenrechte - von 1999..$^{56}$

Der bedeutendste Schritt aber war die Formulierung der Kopenhagener Kriterien, die der Europäische Rat 1993 zusammen mit der Zusage an die post-sozialistischen Reformländer beschloss. Dies stellt die erstmalige schriftliche Festlegung und damit die ,offizielle' Sichtbarkeit der politischen Bedingungen dar, die als Voraussetzung des Beitritts festgelegt wurden. Die politischen Kriterien umfassen die Verwirklichung von institutioneller Stabilität als Garantie für demokratische und rechtsstaatliche Ordnung, die Wahrung der Menschenrechte sowie die Achtung und den Schutz von Minderheiten. Diese Methode der Konditionalität verkörpert streng genommen keine Innovation. Die Kopenhagener Kriterien waren die konsequente Fortsetzung der in den 1960er Jahren begonnenen Konditionalitätspolitik, die nun der Europäische Rat zum Maßstab erhob, was der Politik einen verbindlichen Charakter verlieh. Die Überprüfung der Kriterien wurde der Kommission übertragen. Das Parlament jedoch blieb - wie bereits zuvor - eine engagierte Beobachterin, die sich weiterhin einmischte. Neu war die enge Beobachtung und Evaluierung der Fortschritte, die ab 1998 in den jährlichen Berichten der Kommission transparent und öffentlich gemacht wurde. Diese Berichte dienten als Beurteilungskriterium für die Kommission und als Grundlage für die Entscheidung von Rat und Europäischem Rat. Mit den Kopenhagener Kriterien ${ }^{57}$ und dem Monitoring fand eine Ausdifferenzierung und Konkretisierung der Bedingungen zur Erfüllung von Menschenrechts- und Demokratiestandards statt. Zugleich wurde bald klar, dass die Kriterien keine finale Festlegung darstellten, sondern angesichts neu auftauchender Problematiken weitere Ausdifferenzierungen vorgenommen wurden.

Dies gilt zum Beispiel für die Achtung der Minderheitenrechte, die in der Konditionalitätspolitik der Europäischen Gemeinschaften bislang nicht formuliert worden war. Dies war eine Reaktion auf die nationalistischen Tendenzen und ethnischen Spannungen in den Reformländern, deren Konfliktpotenzial vor dem Hintergrund des Zerfallsprozesses Jugoslawiens der Union vor Augen geführt wurde. Der Europäische Rat reagierte auf dieses Problem 1993, indem er zum einen in Kopenhagen die Achtung der Minderheitenrechte bei den poli-

56 Erklärung über die Menschenrechte, 1991; Europäische Kommission: Mitteilung über die Berücksichtigung der Wahrung der Grundsätze der Demokratie und der Achtung der Menschenrechte in den Abkommen zwischen der Gemeinschaft und Drittländern, der Europäischen Union, KOM (95) 216; Europäische Kommission: Mitteilung der Kommission an den Rat und das Europäische Parlament. Die Menschenrechte in den Außenbeziehungen der Europäischen Union: Von Rom zu Maastricht und danach, KOM (95) 567; Verordnung (EG) Nr. 975/1999 des Rates vom 29. April 1999 zur Festlegung der Bedingungen für die Durchführung von Maßnahmen auf dem Gebiet der Entwicklungszusammenarbeit, die zu dem allgemeinen Ziel der Fortentwicklung und Festigung der Demokratie und des Rechtsstaats sowie zur Wahrung der Menschenrechte und Grundfreiheiten beitragen, in: Amtsblatt der EU, Nr. L 120 vom 8. Mai 1999, S. 1-7; Verordnung (EG) Nr. 976/1999 des Rates vom 29. April 1999 zur Festlegung der Bedingungen für die Durchführung von anderen als die Entwicklungszusammenarbeit betreffenden Gemeinschaftsmaßnahmen, die im Rahmen der Gemeinschaftspolitik auf dem Gebiet der Zusammenarbeit zu dem allgemeinen Ziel der Fortentwicklung und Festigung der Demokratie und des Rechtsstaats sowie zur Wahrung der Menschenrechte und Grundfreiheiten in Drittländern beitragen, in: Amtsblatt der EU, Nr. L 120 vom 8. Mai 1999, S. 8-14.

57 Hier soll nicht tiefer auf die Kopenhagener Kriterien oder die demokratische Konditionalität im Zuge der Osterweiterung eingegangen werden, da dazu inzwischen genügend Studien vorliegen. Siehe auch Seite 228-232 in diesem Band. 
tischen Kriterien einschloss und zum anderen beschloss, als Gemeinsame Aktion einen Stabilitätspakt auszuarbeiten, ,mit dem die Minderheitenfrage geregelt und die Unverletzlichkeit der Grenzen in höherem Maß gewährleistet werden soll“.58 In dieser gemeinsamen Aktion für einen Stabilitätspakt ,ermutigte ' die Union die teilnehmenden Länder, falls noch nicht geschehen, „Abkommen und Vereinbarungen zu treffen über Zusammenarbeit und gutnachbarliche Beziehungen einschließlich Minderheiten- und Grenzangelegenheiten“.59 Auch in der Agenda $2000^{60}$ sowie in den folgenden Berichten der Kommission figurierte der Punkt Minderheitenrechte als eigenes Kriterium. Des Weiteren ergänzte der Europäische Rat auf dem Gipfel von Madrid (1995) substanzielle Maßstäbe mit dem Hinweis auf effiziente Verwaltungs- und Justizstrukturen und die Notwendigkeit der Implementierung von Gesetzen. ${ }^{61}$

\section{Erneute Zunahme an Förderzielen in Südosteuropa}

Dieses verfeinerte und ausdifferenzierte Instrumentarium, das im Zuge der Osterweiterung erheblich fortentwickelt wurde, wandte die Europäische Union auch auf Südosteuropa an. Der 1999 beschlossene Stabilisierungs- und Assoziierungsprozess (SAP) folgte wiederum dem Typus der Beitrittsassoziierung, indem die Union den Ländern des Westbalkans eine Beitrittsperspektive eröffnete bei gleichzeitiger Verpflichtung auf die Kopenhagener Kriterien. Ab 2004 wurde dann die Heranführung dieser Länder analog zu der Osterweiterung angelegt. Die neue Herausforderung bestand darin, dass nicht - wie in der VisegrádGruppe und den Baltischen Staaten etwa - relativ rasch ein stabiles Institutionengefüge und demokratische Verfassungen verabschiedet waren. Sowohl institutionelle Stabilität als auch Menschenrechtsstandards präsentierten sich weitaus prekärer. Zudem hielt die Tatsache, dass es sich um Nachkriegsgesellschaften handelte, zusätzliche Problematiken bereit. Die Europäische Union formulierte daher neue Bedingungen in den Assoziierungsabkommen mit den Staaten des West-Balkans, die darauf reagierten: zum einen die zivile Kontrolle von Militär und bewaffneten Gruppen, zum anderen die Verfolgung beziehungsweise Kooperation bei der Verfolgung von Kriegsverbrechern und schließlich Aspekte wie die Rückführung von Flüchtlingen und Achtung religiöser Minderheiten. Das interethnische und interreligiöse Zusammenleben, die Versöhnung und Stärkung der Zivilgesellschaft sowie soziale Inklusion erlangten für die Beurteilung der politischen Fortschritte auf dem West-Balkan eine neue Bedeutung.

Die fehlende Zusammenarbeit mit den entsprechenden Tribunalen bei der Verfolgung der Kriegsverbrecher wurde indes bereits mehrfach Anlass für die Verzögerung (respektive Eröffnung der Beitrittsverhandlungen mit Kroatien 2005) oder Aussetzung von Verhandlungen (respektive Stabilisierungs- und Assoziierungsabkommen mit Serbien 2006-2007). Auch im Zuge der Beitrittsverhandlungen mit der Türkei kristallisierten sich besondere Probleme heraus: unter anderem Religionsfreiheit und Gewalt gegen Frauen. Mängel bei der

58 Europäischer Rat: Europäischer Rat von Brüssel - 29. Oktober 1993. Schlussfolgerungen des Vorsitzes, Punkt 2.

59 Gemeinsame Aktion zur Fortsetzung der Gemeinsamen Aktion betr. die Eröffnungskonferenz für den Stabilitätspakt vom 14.06.1994, Anhang: Schlussdokument zur Eröffnungskonferenz über einen Stabilitätspakt für Europa, Teil I. Ziele und Grundsätze der Eröffnungskonferenz über einen Stabilitätspakt für Europa in: Auswärtiges Amt: Gemeinsame Außen- und Sicherheitspolitik der Europäischen Union (GASP). Dokumentation, Bonn 1994, S. 508-514.

60 Europäische Kommission: Agenda 2000 - Teil I: Eine stärkere und erweiterte Union - Teil II: Die Erweiterung der Union - Eine Herausforderung, KOM (1997) 2000.

61 Europäischer Rat: Europäischer Rat Madrid 15.-16. Dezember 1995. Schlussfolgerungen des Vorsitzes, SN 400/95. 
Achtung von Menschen- und Minderheitsrechten führten 2006 zum Einfrieren dreier Kapitel bei den Beitrittsverhandlungen. Das heißt, die Europäische Union griff sowohl bei neuen Beitrittskandidaten - Kroatien, Mazedonien und Türkei - als auch bei den Beitrittsaspiranten - Albanien, Bosnien-Herzegowina, Serbien, Montenegro - auf die bewährten Verfahren und Instrumente der Osterweiterung zurück, hatte aber auf neue Aspekte und Probleme im Bereich Menschenrechte und Stabilisierung demokratischer und rechtsstaatlicher Institutionen zu reagieren, was zu einer Erweiterung der anvisierten Förderziele führte.

Der Vergleich der Praktiken der Europäischen Gemeinschaften bei der Süd-, Ost- und Südosterweiterung (Balkan, Türkei) weist klare Analogien auf, aber auch deutliche Unterschiede. So genügte der Gemeinschaft bei den südeuropäischen Ländern die Verabschiedung einer demokratischen Verfassung sowie Wahlen, bei der Osterweiterung und später war dies eine Voraussetzung. Im Zuge der Vorbereitung der Osterweiterung und der dabei auftretenden kritischen Aspekte wurden auch die politischen Kriterien erweitert, veranlasst durch ,neue' Herausforderungen, die auf die Union trafen. Daher ist festzustellen, dass die politischen Kriterien reaktiv angesichts der angetroffenen Problemlagen spezifiziert wurden. Dieses inkrementale Muster, das sich zwischen Birkelbach-Bericht und Kopenhagener Kriterien gezeigt hatte, weist seither eine erhebliche Beschleunigung und Ausdifferenzierung bei der Formulierung politischer Kriterien auf. Das heißt, auch die Kopenhagener Kriterien stellen keinen finalen Maßstab dar.

Der Blick auf die Wegmarken der Erweiterungsdebatte der 1960er Jahre und die Erweiterungsrunden seit den 1980er Jahren hat maßgeblich zweierlei gezeigt: Zum einen, dass die Konditionalitätspolitik eine Kontinuitätslinie verkörpert, und zum anderen, dass die Demokratisierung der sozialistischen Staaten und die Osterweiterungen von 2004 und 2007 ungleich stärker als die anderen beiden analysierten Wegmarken eine Dynamik in der Weiterentwicklung der Menschenrechts- und Demokratisierungspolitik ausgelöst haben. Nachdem in den 1990er Jahren sukzessive Schritte bei der Verankerung von Menschenrechts- und Demokratieprinzipien getan worden waren, stand das folgende Jahrzehnt dann in dem Bemühen, diese Maßnahmen und Instrumente zu einem kohärenteren und konsistenteren Ansatz zuzuschneiden. Ziel sollte ein „Mainstreaming“ von Menschenrechten und Demokratie im gesamten Außenhandeln der Europäischen Union sein. ${ }^{62}$ So wurden 2006 alle bestehenden Finanzierungsinstrumente neu geordnet, um weltweit Menschenrechte und Demokratie zu fördern. ${ }^{63}$ Die Frage, die im folgenden Kapitel diskutiert wird, ist, ob und inwieweit diese angestrebte Konsistenz und Kohärenz sich erreichen lassen angesichts der spezifischen Charakteristika der europäischen Menschenrechts- und Demokratisierungspolitik.

\section{Perspektiven der Menschenrechts- und Demokratisierungspolitik: auf dem Weg zu Kohärenz und Konsistenz?}

Der Beitrag wollte die bislang stark auf die 1990er und folgenden Jahre konzentrierte Perspektive auf die europäische Menschenrechts- und Demokratisierungspolitik um die Betrachtung der vorherigen Jahrzehnte erweitern. Ziel war, eine Entwicklungslogik nachzuweisen, bei der die Menschenrechts- und Demokratisierungspolitik durch externe Herausfor-

62 Europäische Kommission: Mitteilung der Kommission an den Rat und das Europäische Parlament. Die Rolle der Europäischen Union bei der Förderung der Menschenrechte und der Demokratisierung in Drittländern, KOM (2001) 252.

63 Verordnung (EG) Nr. 1889/2006 des Europäischen Parlaments und des Rates vom 20. Dezember 2006 zur Einführung eines Finanzierungsinstruments für die weltweite Förderung der Demokratie und der Menschenrechte - (Europäisches Instrument für Demokratie und Menschenrechte), in: Amtsblatt der EU, Nr. L 386 vom 29. Dezember 2006, S. 1-11. 
derungen im Rahmen der Erweiterungspolitik induziert wurde. Anhand der identifizierten Induktionspunkte konnte gezeigt werden, dass in der Tat die Schübe in der Formulierung von Standards und in der Fortentwicklung der Konditionalitätspolitik zusammenfallen mit den Erweiterungen beziehungsweise Erweiterungsvorbereitungen von vormals autokratischen Regimen, die Demokratisierung und Beitritt zur Gemeinschaft/Union anstrebten. Eine zentrale Rolle für die Entstehung der Methode der Konditionalität spielte die gemeinschaftsinterne Debatte Anfang der 1960er Jahre und der Birkelbach-Bericht, der daher als Keimzelle bezeichnet werden kann. Hier ist zugleich der Anfang der dichotomen Entwicklung anzusetzen in der Festlegung und Anwendung von Menschenrechts- und Demokratiestandards von Ländern mit Beitrittspotenzial einerseits und außereuropäischen Partnerländern andererseits.

Erweiterung beziehungsweise Beitrittsassoziierung wurde durch die Akteure sowohl auf europäischer wie auf nationaler Ebene in den ersten Jahren nach der Gründung der Europäischen Wirtschaftsgemeinschaft nicht als Außenpolitik im klassischen Sinne perzipiert, sondern als zentrales Element des europäischen Einigungswerkes. Mit der Partnerschaftsassoziierung wurde ebenfalls ein nicht klassisches außenpolitisches Instrument geschaffen, bei dem jedoch politische Kriterien (zunächst) keine Rolle spielten. In den Assoziierungsverträgen mit den AKP-Staaten wurde erstmals 1984 (Lomé III-Abkommen) auf Menschenrechte verwiesen; in dem Lomé IV-Abkommen wurden Verpflichtungen der Partner formuliert, der Folgevertrag 1995 enthielt Suspendierungsklauseln und verankerte zudem Menschenrechte, Demokratie und Rechtsstaatlichkeit als wesentliche Elemente. Erst 2000 aber wurden im Cotonou-Abkommen dann ,harte' Klauseln ergänzt und vor allem die Mechanismen bei Nichtbeachtung konkretisiert. Auch die Partnerschaftsassoziierungen mit den Mittelmeerländern, denen teils Assoziierungsverträge, teils Kooperations- oder Präferenzabkommen in den 1970er Jahren vorausgingen, ${ }^{64}$ enthielten erst im Rahmen der Euro-Mediterranen Partnerschaft (Barcelona-Prozess) ab 1995 eine politische Dimension. In den bereits erwähnten Europa-Abkommen mit den ostmitteleuropäischen Staaten und bei der Europäischen Nachbarschaftspolitik (ENP) waren diese von Anfang an eingebaut.

Für beide Entwicklungslinien - Erweiterung und Beitrittsassoziierung hier und Partnerschaftsassoziierung dort - gilt, dass sie nicht auf einem Konzept, einem Masterplan beruhten, sondern in einer inkrementalen Logik als reaktive Antwort auf von außen kommende Herausforderungen vorangetrieben wurden. Die zweite Entwicklungslinie, die hier nicht dargestellt wurde, setzte erst viel später ein. Somit lässt sich in der Menschenrechts- und Demokratisierungspolitik nicht nur eine Dichotomie, sondern auch eine Ungleichzeitigkeit feststellen. Die Keimzelle dieser Dichotomie liegt in der unterschiedlichen Bedeutung, die die Gemeinschaft ursprünglich der Erfüllung von Menschenrechts- und Demokratiestandards gemäß ihres Aktionsfeldes zumaß: Bei den Staaten, die entweder als Mitglieder aufgenommen oder in einer besonderen Form der Assoziierung an die Europäischen Gemeinschaften gebunden werden sollen, wurden immer vergleichsweise höhere Maßstäbe angelegt. Zugleich ist die Angebotsseite jeweils unterschiedlich; das heißt, das höchstwertige Angebot, die Mitgliedschaft, enthält auch den stärksten Hebel. Die jüngere Literatur zur Konditionalität hat diese ,leverage“ als zentrales Element der Erweiterungspolitik identifiziert. Demnach wäre es folgerichtig, wenn die in den Partnerschaftsassoziierungen eingebaute Konditionalität ohne Beitrittsperspektive - wie bei der ENP - eine andere, nämlich

64 Entwicklungsassoziierungen wurden mit ausgewählten Mittelmeerländern in den 1970er Jahren abgeschlossen (1969: Marokko, Tunesien; 1970: Malta; 1972: Zypern), Kooperationsabkommen mit Israel (1970) und den Mashrik-Staaten (1977). 
schwächere Hebelwirkung entfaltet. Erste empirische Studien stellen dies fest, ${ }^{65}$ es bedarf hierzu aber weiterer, vergleichender Untersuchungen.

Eine zweite Frage dieses Beitrags war, inwiefern der neo-funktionalistische Ansatz der Innen-Außen-Analogie auch in der historisch-genetischen Sichtweise Erklärungskraft beweist. Hier müssen zwei Modifizierungen vorgenommen werden: Zum einen belegt die lange Zeit vorherrschende Dichotomie zwischen europäischen und außereuropäischen Partnern, dass die Gemeinschaft bei der Externalisierung ihres Wertekerns regionale Abstufungen vornahm. Diese Abstufungen (siehe Abbildung 1) unterstreichen, dass das Verständnis der Europäischen Union als Förderin von Menschenrechten und Demokratie originär mit ihrem regionalen Ordnungsanspruch - oder anders gesagt: mit dem europäischen Einigungsgedanken - verknüpft war und dies im Zuge der Entwicklung dieser ,policy “ weitgehend auch blieb. Die Europäische Union hat sich als regionale Ordnungsmacht etabliert und auch profiliert und in ihrem regionalen Einzugsbereich ist sie zweifelsohne eine Exporteurin von demokratischen und rechtsstaatlichen Standards und Werten geworden, wobei sie in einem graduellen Prozess ihr Selbstbild einer demokratischen Wertegemeinschaft schärfte. Mit dieser regionalen Abstufung korreliert zudem auch die unterschiedliche Hebelwirkung der europäischen Konditionalitätspolitik.

\section{Abbildung 1: Regionale Abstufung und Abstufung des Konditionalitätshebels}

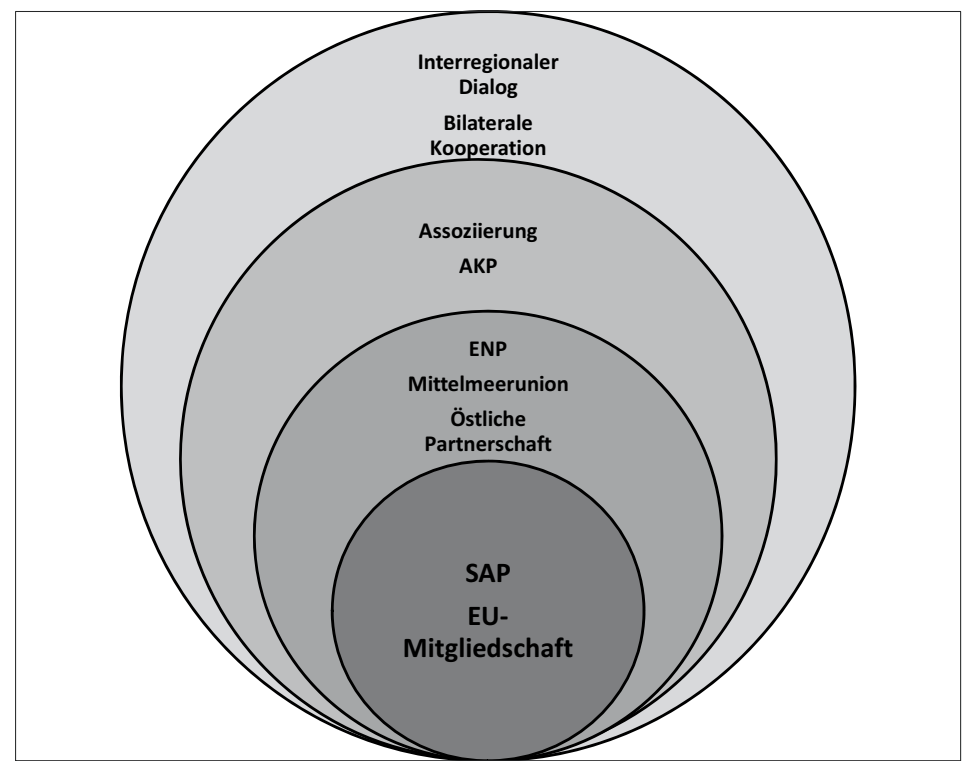

Quelle: Eigene Darstellung.

Die zweite Modifizierung der Innen-Außen-Analogie lautet: Der interne, gemeinschaftsimmanente Konsens über die Wertebasis und demokratische Verfahren und Prinzipien generierte tatsächlich - der Externalisierungslogik folgend - ein Politikfeld; dieses wirkte aller-

65 Annette Jünemann: Realpolitisches Nutzenkalkül oder konstruktivistischer Rollenkonflikt? Erklärungsansätze für die Inkohärenz europäischer Demokratieförderung im südlichen Mittelmeerraum, in: Knodt/Jünemann: Europäische Demokratieförderung, 2007, S. 295-317; Annegret Bendiek: Wie effektiv ist die Europäische Nachbarschaftspolitik?, 2008, S. 9. 
dings stärker nach außen, während es im Inneren lange keine ,policy'-Entsprechung fand. Der Profilierung als Exporteurin demokratischer Werte und Prinzipien stand lange kein entsprechendes Politikfeld im Inneren komplementär gegenüber. Mit dem Inkrafttreten des Vertrags von Lissabon wird für die Konzeption der Menschenrechts- und Demokratisierungspolitik eine neue qualitative Stufe erreicht, denn die immer wieder konstatierte Kluft zwischen interner und externer Dimension konnte - formell - erst mit der Inkorporierung der Grundrechtecharta geschlossen werden. Ob damit auch jene Ungleichzeitigkeit zwischen Werteexport nach außen und den hinterherhinkenden inneren Entwicklungen ausgeglichen werden kann, wird sich zeigen müssen. ${ }^{66}$ Es muss erst beobachtet werden, wie sich die Neuerungen des Vertrags von Lissabon auswirken werden und wie etwa die Inkorporierung der Grundrechtecharta und das Wegfallen der Pfeiler diese Inkohärenz zwischen innerer und äußerer Menschenrechts- und Demokratisierungspolitik verändern werden.

Mit dem , globalen ‘ Ansatz, den die Europäische Union in ihrer Menschenrechts- und Demokratisierungspolitik seit den 1990er Jahren vertritt, hat sie jedenfalls die Ansprüche erhöht. Von einer neuen, europäischen Menschenrechtspolitik wird im Kern erwartet, dass sie (1) die immer wieder geforderte, umfassende und kohärente' Strategie entwickelt, (2) die Verbindung zwischen internen und externen Politiken sowie eine stärkere Interaktion und Komplementarität der beiden Ebenen fördert, (3) die Koordination zwischen den zahlreichen Gemeinschaftsprogrammen effektiver koordiniert sowie (4) auch die Menschenrechtsdimensionen der drei Pfeiler enger abstimmt und in Einklang bringt. ${ }^{67}$ Im letzten Jahrzehnt wurde die vermehrte Tätigkeit der Organe zur Weiterentwicklung der Menschenrechts- und Demokratisierungspolitik angeleitet von dem Ziel höherer Kohärenz und Konsistenz; und zwar sowohl in Bezug auf die Anwendung der Standards als auch in Bezug auf die Instrumente. Dennoch werden genau diese Aspekte weiterhin kritisiert. Eine jüngere Studie des Parlaments weist auf die Inkonsistenzen zwischen interner und externer Dimension der Menschenrechtspolitik hin. Zudem wird eine systematischere Herangehensweise eingefordert. ${ }^{6}$

Konsistenz bleibt also ein Desiderat. Ob solch ein konsistenter Ansatz erreicht werden kann, ist jedoch fraglich. Die Genese und Entwicklungslogik der Menschenrechts- und Demokratisierungspolitik haben deutlich gemacht, dass dieses Politikfeld durch zwei sehr früh herausgebildete Binome - erstens: innere und äußere Dimension der Menschenrechts- und Demokratisierungspolitik; und zweitens: Länder mit versus Länder ohne Beitrittspotenzial geprägt ist. Damit sind zugleich Gründe (freilich nicht die einzigen!) für Unterschiede vor allem in der Anwendung herausgearbeitet. Dies widerlegt auch die , one size fits it all'These ${ }^{69}$, denn es ist nicht so, dass die Europäische Union eine identische Schablone auf jedes Land legt und diese mit dem gleichen Instrumentarium abarbeitet; sei es wegen der genannten Inkonsistenzen im Ansatz oder sei es, weil sie sich an den konkreten Rahmenbedingungen im Adressatenland orientiert.

Die historisch-genetische Betrachtungsweise hat sich als lohnend erwiesen, um Erklärungsmomente für den bisherigen Zuschnitt der europäischen Menschenrechts- und Demo-

66 Vgl. Williams: EU Human Rights Policies, 2004, S. 79-111; sowie Andrea Ott: The EU and Human Rights. Leading by Example?, in: Apirat Petchsiri/José Luis de Sales Marques/William Roth (Hrsg.): Promoting Human Rights in Asia and Europe. The Role of Regional Integration, Baden-Baden 2009, S. 83-99.

67 Vgl. Alston/Weiler: An ,Ever closer Union', 1999.

68 Vgl. European Parliament. Directorate-General for External Policies. Policy Department: Human Rights Mainstreaming in EU's External Relations. Study, EXPO/B/DROI/2008/66, September 2009, S. 46ff.

69 Vgl. Tanja Börzel/Thomas Risse: One Size Fits All! EU Policies for the Promotion of Human Rights, Democracy and the Rule of Law. Workshop on Democracy Promotion, Stanford University 2004. 
kratisierungspolitik aufzutun. Und es spricht Einiges dafür, dass auf dieser Grundlage auch Aussagen über die Prägung der künftigen Entwicklung gemacht werden können. Auch wenn das Politikfeld Ergänzungen und Verfeinerungen erfahren hat, ist die Handlungslogik weiterhin von Dichotomien geprägt und wird es mit großer Wahrscheinlichkeit bleiben. Insofern fällt die Prognose eher skeptisch aus, was eine künftige Steigerung der Kohärenz angeht.

\section{EU-Komissare}

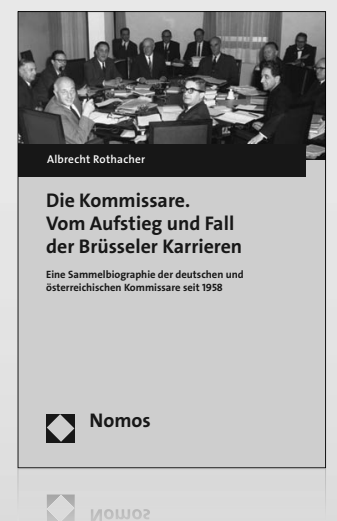

Weitere Informationen: www.nomos-shop.de/14228

\section{Die Kommissare. Vom Aufstieg} und Fall der Brüsseler Karrieren

Eine Sammelbiographie der deutschen und österreichischen Kommissare seit 1958

Von Dr. Albrecht Rothacher

2011, ca. 245 S., brosch., ca. 44,- $€$

ISBN 978-3-8329-7097-0

Erscheint ca. Dezember 2011

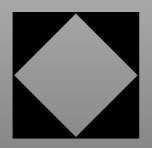

Nomos 